Vito Gentile, Passant Elagroudy and Ecem Ergin Pervasive Displays Research: What's Next? In IEEE Pervasive Computing, vol. 18, no. 1, pp. 72-78 2019 


\title{
Pervasive Displays Research: What's Next?
}

\author{
Vito Gentile \\ University of Palermo \\ Passant Elagroudy \\ University of Stuttgart \\ Ecem Ergin \\ University College London \\ Department Editors: \\ Sarah Clinch \\ sarah.clinch@manchester.ac. \\ uk
} emos. The general chair, Albrecht Schmidt from the Ludwig-Maximilians-Universität München (LMU), and the program co-chairs, Julie Rico Williamson (University of Glasgow) and Ivan Elhart (Università della Svizzera Italiana, USI), opened the conference by reporting the statistics of PerDis 2018. This year, the conference attracted 44 attendees from 10 different countries, showing a promising growing trend if compared with last year edition. PerDis strives to create a compelling environment for young and seasoned researchers alike. Thus, it recognizes outstanding contributions using awards for the best paper, demo and poster via attendees voting.

\section{RESEARCH TRENDS}

The conference programme included four paper sessions, namely: "Large Displays and Design Strategies," "Display Applications and Interfaces," "Novel Displays and Installations," and "Augmented Reality, Virtual Reality and Display Awareness." During the conference, 22 full papers were presented. Additionally, nine posters and four demos were presented in a subsequent session. Here, the contributions are clustered into thematic areas in order to highlight the main trends in pervasive displays research, which have been also summarized in Figure 1.

\section{Application Areas for Pervasive Displays}

Nine contributions presented applications for using displays. Wearable displays such as Augmented Reality (AR) glasses, Head-Mounted-Displays (HMDs), Virtual reality (VR) sets and vibrotactile bands had the highest research momentum (four papers), followed by ambient displays such as wall tablets (two papers and one poster), and public displays (two papers and one poster). 


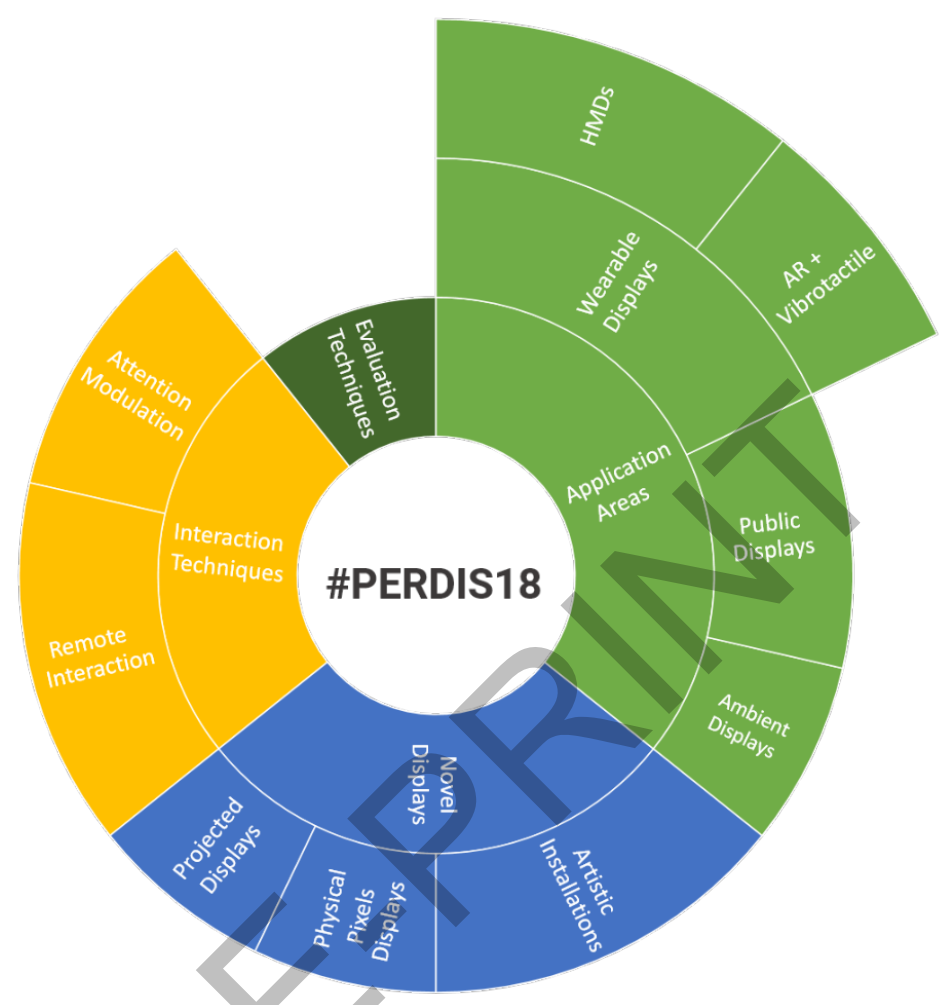

Figure 1. Emerging themes of pervasive display research in PerDis 2018. Most of the contributions were focused on application areas for pervasive displays with a rise in the submissions related to wearable displays (e.g. AR/VR applications). Several papers focused also on novel displays, such as those based on levitating particles and plant-based displays.

\section{Public Displays}

An emerging theme in pervasive displays research was how to design applications for networked displays. It is no surprise that during PerDis 2018 this theme has been covered by a couple of papers. Mateusz Mikusz (Lancaster University) presented his work on the use of a network of pervasive displays aimed at aiding student recall in an academic campus. A longitudinal study has been conducted in a campus-wide trial to investigate how showing lecture slides as memory cues may support computer science students. After-presentation discussions focused on the research challenge of efficient content creation and cue selection. Examples include content creation responsibility and whether it belongs to the instructors, or the students and whether the objective of the screens is to help students remember vs. understand the content better. In a poster presentation, Peter Shaw (Lancaster University) introduced technical details about the mobile application used in the trial to improve the interaction.

Similarly, Rui José (University of Minho) et al. used a network of pervasive displays, but in a different context. They explored the use of networked LED message sign displays in the urban context. José provided an overview of the possible design challenges that future work will face. These include the need for supporting interaction with networks of displays, as well as issues with infrastructure, dealing with integration in the existing architecture, and choices about content. Scalability and applicability of such networks to different contexts was also proposed as an additional issue to be considered in future designs. 


\section{Ambient Displays}

Two papers focused on exploring ambient displays in a domestic context. The first paper by Marius Hoggenmueller (LMU Munich) presented an exploratory case study on the use of low-resolution lighting displays aimed at visualizing domestic energy performance data. Three displays were deployed in three different households, and then the families were interviewed. Hoggenmueller explained that using such displays can be preferred to more traditional mobile applications (e.g., via tablets). Showing live data may gain user acceptance more easily than visualizing historical data on mobile devices.

The second paper by Alexandra Voit (University of Stuttgart) et al. introduced Caloo, an ambient and pervasive smart wall calendar with event suggestions. Caloo aims to increase users' awareness about upcoming events, also engaging them to suggest additional events. Voit presented the outcome of an evaluation study of Caloo, suggesting that users value the interactive calendar and are eager to use it. Insight on future work included comparing this approach to using projectors and investigating the impact of illumination levels on the user experience.

\section{Wearable Displays}

HMDs, arm bands and AR-oriented wearable markers were explored by several papers this year. In particular, Liviu Coconu (neARtracker) and Brygg Ullmer (Clemson University) presented an interesting demo for interacting with a virtual garden using a tangible device and a HMD. This demo showcased the apparatus described in this year's best paper award winner by Ullmer et al., which covered a project originally deployed in 2004 . After 14 years, the project came back to life with low-cost technologies, using an inexpensive tracking sensor mounted on a smartphone to bring together tangible interaction and virtual reality. The talk, along with the subsequent discussion, offered some hints on how this project revival may occur again in the future $(2032,2202)$ using new technologies such as HMDs or BCI.

The theme of combining modalities for displaying and modelling content was also explored by Tim Claudius Stratmann (University of Oldenburg), who explored the use of wearable and vibrotactile displays (i.e., AR glasses and armbands) to spatially guide users with vibrations and peripheral visual cues. Stratmann explained that both visual and vibrotactile cues successfully guide the users, but using both simultaneously increases the workload and should be avoided. Vibrotactile wearable displays have also been explored by Vanessa Cobus (OFFIS Institute for IT) et al., in order to help in delivering efficient alarms to nurses, reducing acoustic noise. She addressed the problem of alarm-fatigue resulting from conflicting noise as it causes a delayed or inadequate response time to crucial alarms. She showed that a vibrotactile wearable solution is suitable because it provides low error rates, short response times, and good usability. The discussion that followed the talk focused on potential technical developments of the prototype, such as increasing battery duration and managing thresholds for better deciding when to deliver the alarms. Cobus also demoed her system in the conference.

Aesthetic and ubiquitous displays were explored by Ashley Colley (University of Lapland), who introduced the concept of "Smart Jewelry." Colley and his group proposed the use of jewels as AR markers, thus allowing for displaying information via mobile applications. They described the process of making jewels, and which characteristics make them compliant with AR-oriented interactions.

\section{Novel Displays}

Nine contributions presented novel displays. The contributions are clustered around three categories: 1) displays using projections in novel locations, 2) displays using physical interactive objects as their pixels, and 3 ) environmental installations re-purposed as displays.

\section{Projected Displays}

Two papers focused on exploring the car as a medium for displaying information. The first paper by Ashley Colley (University of Lapland) et al. investigated the use of displays on exterior surfaces 
of cars. Colley and his colleagues conducted a focus group with projected displays to understand users' preferences and feedback. They showed that solutions focusing on improving safety are considered most beneficial, and that displaying individually targeted information was mostly disliked due to privacy concerns. The second paper by Andreas Riegler (University of Applied Sciences Upper Austria) et al. discussed an investigation of autonomous vehicles' users about the use of windshield displays. He showed which areas of windshield displays are considered most important by potential users, and the importance of adaptive content based on the level of automation. For instance, warning messages are considered more important when take-over situations are expected, while infotainment systems are preferred when a higher level of automation is provided by the car. The subsequent discussion focused on the content selection, suggesting the importance of investigating aspects such as users' responses to advertisements.

Anke M. Brock (University of Toulouse) and Jessica R. Cauchard (IDC Herzliya) used a different medium and introduced "FlyMap" in their paper, an investigation on how to interact with displays projected from drones. Envisioning the use of such drones in aid scenarios, they conducted two pilot studies aimed at investigating interactions via smartphone and mid-air gestures with maps. The discussion focused on proposing alternative interaction methods such as voice control and highlighted the importance of investigating the affordance blindness problem (i.e., users' inability to understand the interaction modality supported by the display).

\section{Artistic Installations}

This set of contributions focused on repurposing existing establishments in the environment to pervasive displays by introducing new interactions with them. For example, Antti-Jussi Yliharju (University of Lapland) et al. explored the use of ice as an interactive visualization. Yliharju provided an overview of ice characteristics, and how to exploit them in conjunction with controllable lights. He also presented a design space for ice as a design material, particularly as part of interactive systems, based on examples and domains where ice has been utilized. This novel idea fostered an interesting discussion on using ice as a visualization medium to connect cold places with hot places, and how technologies like thermal cameras may be helpful in enabling vision-based interactive capabilities. He also gave a poster presentation showing a snow wall installation as a novel display material for interactive public experience, based on the use of mid-air gestures. Another idea, presented by Ecem Ergin (UCL), was the utilization of orange robotic arms, usually adopted for automatizing assembly lines, as a new form of public display. Ergin showed how these kinetic installations (which included also light sources, mirrors and soundscapes) functioned as a socializing platform. Thus, they can also be approached as a new typology of media interfaces embedded in the everyday city environment, pointing to a new potential application of urban human-computer interaction. Moving from large scale installations to personal and portable ones, Beat Rossmy (LMU Munich) presented a modular, low-resolution display to support electronic musical pre-education called COMB. This poster presentation discussed designing meaningful interaction using the low-resolution display that allowed user to choose different instruments by changing the display layout.

\section{Physical Pixels Displays}

During the conference, several presentations focused on physical pixels, i.e., objects used in groups as a display to mimic digital pixels. In particular, the conference programme included papers showing airborne vs. grounded physical pixels. Julie R. Williamson (University of Glasgow) presented her group's work on levitating particle displays, using ultrasound to allow physical "particles" (voxels) to be positioned and controlled in mid-air. Williamson proposed a design space and challenges for interacting with levitating displays and using them in order to visualize information. Levitating displays elicited an interesting discussion about supporting voxels of different shapes and sizes, as well as using different physical properties (e.g., magnetic fields) to overcome some of the limitations of using ultrasound. On the other hand, Vito Gentile (University of Palermo) introduced Plantxel, the first prototype of a plant-based pixel to be used in a wider plant-based controllable display. He described a feasibility study, showing that the use of thigmonastic plants (i.e., plants that respond to external stimuli such as touches or vibrations) allows for good contrast 
ratios. The discussion focused on the challenges of transporting the plants as well as maintaining them, as compared to controlling them.

\section{Interaction Techniques for Pervasive Displays}

Eight contributions focused on interactions. The contributions can be clustered into two categories: 1) remote interaction, 2) techniques for easing interactions.

\section{Remote Interaction Techniques}

There was a trend focusing on mid-air and touchless interactions this year in the presented work (two papers and one poster). Florian Alt (LMU Munich) introduced ShapelineGuide, a dynamic visual solution to guide users of large interactive displays in performing the correct mid-air gestures. ShapelineGuide includes a feedforward system that shows the possible 3D paths to perform all the supported gestures. Brock et al. also explored mid-air gestures for drone projections, as discussed earlier. Similarly, Vito Gentile (University of Palermo) used touchless interaction as well. Gentile et al. created an avatar-based interface to allow autistic users to access information about artwork. An exploratory study suggested that touchless interaction can increase usability and accessibility. On the other hand, Jake Patterson (University of Manchester) focused on using a mobile phone to support remote interaction. In his paper, he proposes "SlideTalk," a tool allowing an audience to comment and control slideshows using personal mobile devices. Patterson discussed the results of a comparison study, showing a higher number of interactions when users were able to comment on the display contents, suggesting that SlideTalk was able to foster engagement with displays. The subsequent discussion involved many questions about the benefits of including, or not, comments on the display, how to manage the pagination, and how this solution can be ported to any other display system.

\section{Techniques for Easing Interactions}

The following contributions focus on drawing the attention of users to the presence of the displays or their content. For instance, Salvatore Sorce (University of Palermo) presented a poster that investigates a sound-based solution for drawing acoustic paths towards an interactive public display, in order to use it for overcoming display blindness. Marius Hoggenmueller (LMU Munich) discussed how to draw attention to common failures affecting public interactive displays. He proposed five design strategies aimed at overcoming the common software, hardware and environmental issues. Complementarily, Uwe Gruenefeld (University of Oldenburg) explored the AR realm in the context of pervasive display research, presenting FlyingARrow. This solution aims at guiding users to locate and visualize out-of-field objects, a common issue in many AR and VR applications. The results showed that FlyingARrow performs better than previous work. He also demoed "EyeSeeX," a visualization technique aimed at guiding users to visualizing out of view objects in VR.

\section{Evaluation Techniques for Pervasive Displays}

Three papers were presented investigating the impact of external factors on classical evaluation methods or proposing the usage of existing models in other domains. Patrick Tobias Fischer (Bauhaus-Universität Weimar) et al. investigated the temporal, spatial and contextual impact on users' ratings when using AttrakDiff, a standard questionnaire commonly used to evaluate the user experience in response to interactive public installations, media façades and public displays.

Two papers borrowed from existing metric models and used them across domains. The first paper, by Jim Wallace (University of Waterloo) et al., was about evaluating a user's fatigue while interacting with large multi-touch displays. Wallace showed how it is possible to apply the Cumulative Fatigue (CF) model, originally designed for mid-air gestures, in order to make it usable with multitouch interactions. In the subsequent discussion, Wallace explained that the model can be calibrated to achieve error rates comparable to those for mid-air interaction, and that this model can 
potentially be applied to single-touch interactions. On the other hand, Christian Mai (LMU Munich) investigated the use Head-Mounted-Displays (HMDs) in public settings. Usually, HMDs require the presence of some operators who help users to wear them. However, this research models the behavior of public HMDs in a way that resembles the well-known audience models defined in prior pervasive display research. This approach elicited many questions from the audience, including how to evaluate the affordances of HMDs, and if this model can be applied to other fixed large displays for exploring $3 \mathrm{D}$ environments.

\section{Miscellaneous Themes}

Education was an emerging theme that included Mikusz's paper about a campus trial for memory augmentation using lecture slides, and two additional poster presentations. The first, by Matthias Ehlenz (RWTH Aachen University), presented a framework for multi-touch learning games that incorporates learning analytics components and tangibles to improve collaborative learning experiences. The second, by Jonas Herbert (Ruhr University of Bochum), defined a framework for documenting Lego Serious Play (LSP), based on the use of Hololens in AR. The system was also demoed. Complementarily, Jakob Karolus (Technical University of Darmstadt) investigated proficiency-aware systems that can sense user's proficiency to adapt the presentation of content. Similarly, Calvin Liang (Tufts University) explained how eye tracking can be used as a real-time method for evaluating programming skills.

Collaborative interactions also interested some contributors to the poster track. Yashio Matsuda (Saitama University) focused on a user interface that allows multiple users to interact simultaneously and obtain information from a public display. On the other hand, Masahumi Muta (University of Tsukuba) explored how to reduce social awkwardness from interactions by providing an interface for a non-human fitting room experience in fashion, in order to reduce stress of speaking out one's true opinions and suggestions.

\section{EMERGING THEMES IN PERVASIVE DISPLAYS RESEARCH \\ Emerging Themes from the Keynotes}

The two keynotes had a complementary nature this year. Prof. Nigel Davies (Lancaster University) focused on sketching a solid research agenda for public displays research and Prof. Gudrun Klinker (Technical University Munich) presented her vision about human-machine partnership where intelligence amplification supersedes artificial intelligence.

In the opening keynote, Prof. Davies highlighted the need for keeping a critical eye on "saturated display environments" where being surrounded by numerous screens is the default. Setups like Times Square in New York or Piccadilly Circus in London, with dozens or hundreds of screens, arise a plethora of new research questions. For instance, new effective interaction techniques are required, as well as effective ways to engage users with content, despite the cognitive overload due to the high number of screens. New engagement metrics are also required, since number of passers-by or glances at a screen become obsolete in this context. Even phenomena like display blindness need to be re-analyzed almost from scratch. Prof. Davies highlighted that we do not know yet how to identify the optimal number of screens to cover a certain space, in order to make the content engaging for the highest possible number of passers-by, while minimizing costs of installations, maintenance and energy consumption. All these ideas represent low hanging fruits for the pervasive displays community, in order to create interesting and impactful research in the current evolving contexts.

In the closing keynote, Prof. Klinker proposed the use of gamification and augmented reality as strategies for increasing human motivation, and thus efficient communication with the machines to leverage intelligence. She discussed the impact of gamification on various user groups, such as motivating behavioural change in older adults, or increasing the productivity of workers. While 
worker resisted serious games because "they are not here to play," older adults developed a dependency on the games that enabled the caretakers to use them to motivate behavioural change. Prof. Klinker closed by promoting "Gamified Steerable Simulation Environments," supporting multiple displays and ubiquitous interactions.

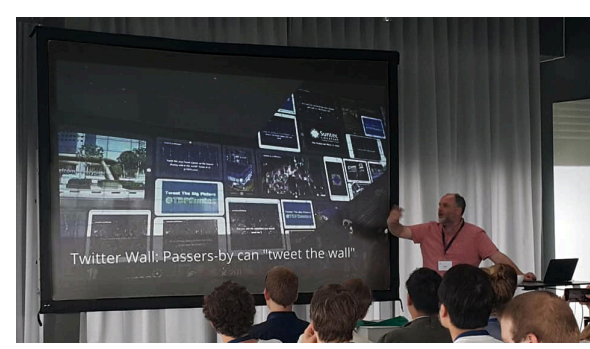

(a)

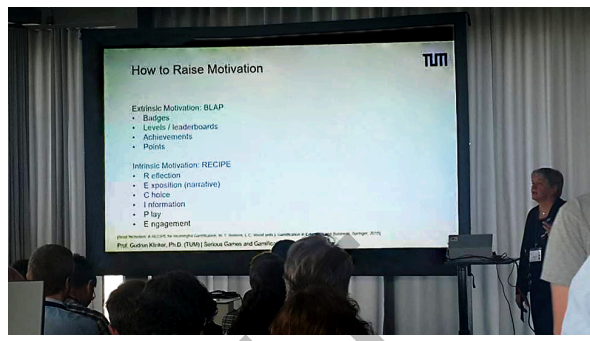

(b)

Figure 2. The two keynote speakers at PerDis 2018. In (a), Prof. Nigel Davies presented a keynote on saturated display environments, and in (b), Prof. Gudrun Klinker's keynote was about serious games and gamification for augmented reality.

\section{Emerging Themes from the Conference}

Looking at the previous PerDis conferences, some trends have been confirmed, but new ones emerged. In particular, focusing on PerDis 2016 and 2017, we can see an increasing interest towards AR and VR. Both themes were discussed in PerDis 2016 (where a tutorial was dedicated to VR), but PerDis 2018 had entire conference session dedicated to this topic.

Other themes still continue to be of interest, such as interaction techniques and the need for supporting immediate usability, or allowing multi-user interactions. Other themes are novel display hardware and technologies, understanding audience behavior, techniques for attracting and engaging users, and case studies in-the-wild.

Interestingly, the 2018 conference had less emphasis on software systems, e.g. operating system for pervasive displays, or novel content-scheduling solutions. An entire conference session was dedicated to this theme in PerDis 2016, and even more papers covered such topics in prior editions. This may be seen as a shift towards HCI themes versus software engineering.

Finally, the three one-day tutorials covered hot research topics in the community to reduce the entry barrier for young researchers. The first one, by Prof. Florian Alt (Bundeswehr University Munich) and Mohamed Khamis (LMU Munich), was about the basics of pervasive display research, covering concepts such as interaction design and evaluation. Reflecting the emerging research trend to use AR and VR technologies in the context of pervasive displays, the second tutorial by Pascal Knierim (LMU Munich) and Matthias Hoppe (LMU Munich) offered an introduction to augmented reality technologies such as Unity and Hololens. The third tutorial by Sven Mayer (University of Stuttgart) and Huy Viet Le (University of Stuttgart) offered an introduction to machine learning techniques to implement intelligent systems.

\section{CONCLUSION}

Prof. Nigel Davies from the steering committee concluded the symposium with an open discussion about the conference, to get attendee feedback and ideas to improve future editions. Participants were also asked to leave feedbacks on Post-It Notes. Reviewing those comments, the main strengths of the conference were reported to be the engaging and competent community, as well as the many opportunities for networking.

In general, the work presented in PerDis 2018 reflects the most recent advances in pervasive displays research. The interest from the community, and the new frontiers envisioned, suggest a potential growing interest in these topics, which can provide a great addition to the pervasive computing research area. 
During the conference, next year's general chair Salvatore Sorce announced that PerDis 2019 will be hosted in June in Palermo, Italy. Papers accepted to PerDis 2019 will be invited to extend their work for a special issue on pervasive displays published by the Springer journal "Personal and Ubiquitous Computing."

\section{ABOUT THE AUTHORS}

Vito Gentile is a research fellow at the University of Palermo, Italy. Contact him at vito.gentile@unipa.it.

Passant El.Agroudy is a $\mathrm{PhD}$ student at the University of Stuttgart, Germany. Contact her at passant.el.agroudy@vis.uni-stuttgart.de.

Ecem Ergin is a PhD student at the University College London, UK Contact her at ecem.ergin.17@ucl.ac.uk. 